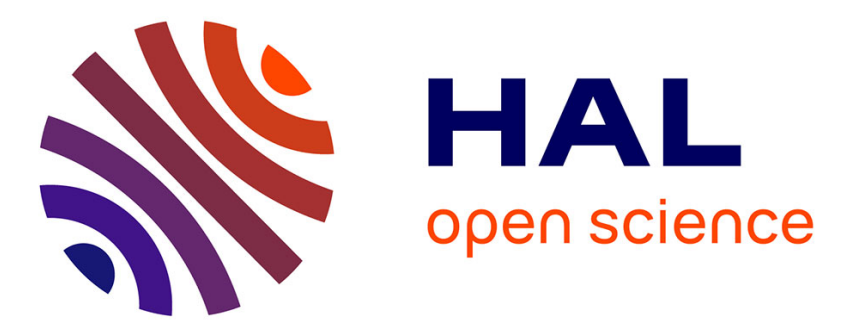

\title{
On the nuclear isovector spin response in the quasielastic peak region
}

U. Stroth, R.W. Hasse, P. Schuck, W.M. Alberico, A. Molinari, M. Ericson

\section{To cite this version:}

U. Stroth, R.W. Hasse, P. Schuck, W.M. Alberico, A. Molinari, et al.. On the nuclear isovector spin response in the quasielastic peak region. Physics Letters B, 1985, 156, pp.291-295. 10.1016/03702693(85)91611-9 . in2p3-00004317

\section{HAL Id: in2p3-00004317 https://hal.in2p3.fr/in2p3-00004317}

Submitted on 23 Jun 2021

HAL is a multi-disciplinary open access archive for the deposit and dissemination of scientific research documents, whether they are published or not. The documents may come from teaching and research institutions in France or abroad, or from public or private research centers.
L'archive ouverte pluridisciplinaire HAL, est destinée au dépôt et à la diffusion de documents scientifiques de niveau recherche, publiés ou non, émanant des établissements d'enseignement et de recherche français ou étrangers, des laboratoires publics ou privés. 


\title{
ON THE NUCLEAR ISOVECTOR SPIN RESPONSE IN THE QUASIELASTIC PEAK REGION ${ }^{\text {मे }}$
}

\author{
U. STROTH, R.W. HASSE \\ Institut Laue-Langevin, F-38042 Grenoble Cedex, France
}

P. SCHUCK

Institut des Sciences Nucleaires, F-38026 Grenoble Cedex, France

W.M. ALBERICO, A. MOLINARI

Istituto di Fisica Teorica, Università di Torino, I-10125 Turin, Italy

and

M. ERICSON

Institut de Physique Nucléaire, Université Lyon-1, F-69622 Villeurbanne Cedex, France and CERN, CH-1211 Geneva, Switzerland

\begin{abstract}
We study the isovector spin responses at medium momentum transfers. We use nonlocal mean field plus standard residual particle-hole interactions to calculate the responses in a semiclassical approach. The data on inelastic electron scattering and the ratio of longitudinal to transverse isovector spin surface response as measured recently by inelastic proton scattering are analysed in a consistent manner.
\end{abstract}

The spin-isospin response function in infinite nuclear matter is expected to show collective effects even at momentum transfers as high as $q \simeq 2 \mathrm{fm}^{-1}$. This prediction is based on nuclear matter calculations using the so-called ring approximation and a ph interaction constructed from pion and rho meson exchanges together with a short range component [1]. At moderate momenta this interaction $[1,2]$ turns out to be attractive in the spin longitudinal channel and repulsive in the spin transverse channel and, thus, the corresponding responses or structure functions $S_{\mathrm{L}}$ and $S_{\mathrm{T}}$ should be enhanced and quenched respectively, compared to their noninteracting values.

In nuclear matter calculations the finiteness of the nucleus and the surface effects are supposed to be ap-

\footnotetext{
This work is part of the Ph.D. thesis of U. Stroth.
}

proximately taken into account through the introduction of an effective adjustable Fermi momentum $k_{\mathrm{F}}^{\text {eff }}<k_{\mathrm{F}}$. The validity of this approach is questionable for light nuclei and also if the response is explored through a strongly interacting probe, such as protons, since then it is the surface response which is involved rather than the volume one (as appears for instance with electron or neutrino probes).

In order to eliminate this type of uncertainty we apply in the present work a simple semiclassical approach to account, in an accurate way, for the finiteness of the system. In a second step we generalise the model of ref. [1] and investigate the influence of the nonlocality of the Hartree-Fock field on the response function. We analyse consistently $\left(e, e^{\prime}\right)$ scattering and the Los Alamos ( $\left.p, p^{\prime}\right)$ experiment [3] on the ratio of the spin longitudinal and trans- 


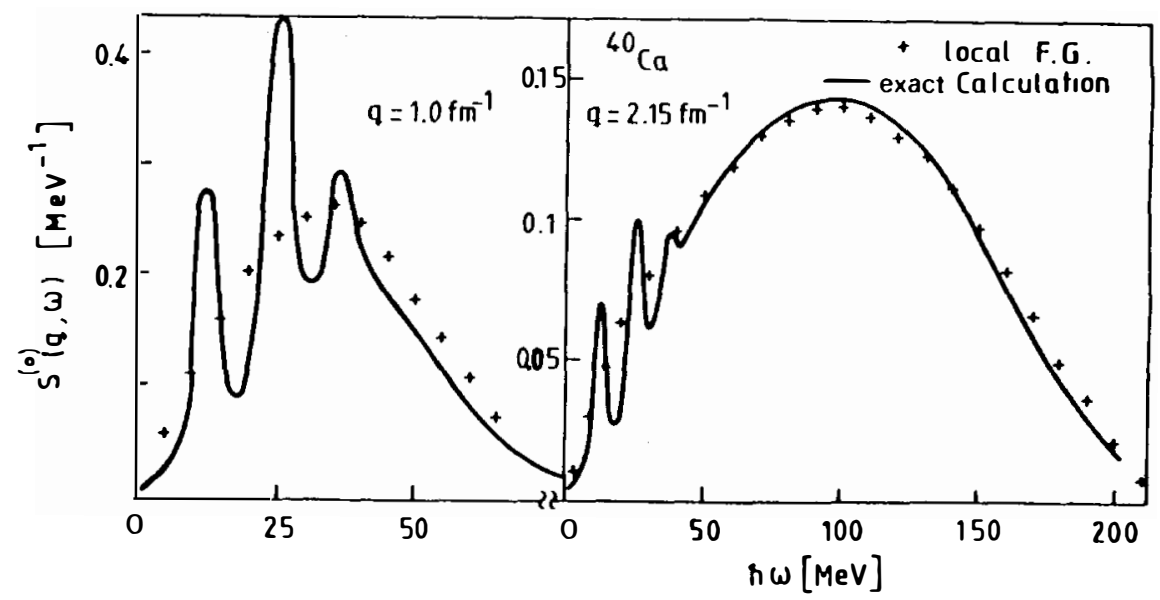

Fig. 1. Semiclassical and exact structure function at two momentum transfers (for details, see text). The exact results have been folded with a lorentzian of $6 \mathrm{MeV}$ width.

verse responses. The absorption of the high energy protons is treated in an eikonal approximation to the optical model wave functions.

The $1 \mathrm{p}-1 \mathrm{~h}$ nuclear response function in ring approximation for spin longitudinal and spin transverse excitation operators $\sigma q \exp (\mathrm{i} q r)$ and $\sigma \times q \exp (\mathrm{i} q r)$, respectively, for finite nuclei has been shown to have the following form to lowest order in $\hbar$ [4]:

$$
\begin{aligned}
& \Pi_{\mathrm{L}, \mathrm{T}}(q, \omega)=4 \pi \\
& \quad \times \int_{0}^{R} \mathrm{c} \mathrm{d} R R^{2} \frac{\Pi^{(0)}(R, q, \omega)}{1-v_{\mathrm{L}, \mathrm{T}}^{\sigma=1, \tau=1}(q, \omega) \Pi^{(0)}(R, q, \omega)} .
\end{aligned}
$$

Here $v_{\mathrm{L}, \mathrm{T}}$ is the ph interaction for which we take the form given in refs. $[1,2] ; \Pi^{(0)}$ is the free (non-interacting) response in the local momentum approximation, i.e. in the nuclear matter expression $k_{\mathrm{F}}$ is replaced by $k_{\mathrm{F}}(R)=\left[2 m\left(\epsilon_{\mathrm{F}}-V(r)\right) / \hbar^{2}\right]^{1 / 2} ; V(R)$ is the local mean field for which we use the parameterisation of ref. [5] and $R_{\mathrm{c}}$ is the classical turning point determined by $\epsilon_{\mathrm{F}}=V\left(R_{\mathrm{c}}\right)$, with $\epsilon_{\mathrm{F}}$ the Fermi energy.

In order to demonstrate the accuracy of the semidassical approximation we calculate [4] the nuclear structure function for noninteracting nucleons in a Woods-Saxon potential with the following values of the parameters (in the usual notation): $V_{0}=-50$ $\mathrm{MeV}, R_{0}=1.2 A^{1 / 3} \mathrm{fm} ; a=0.5 \mathrm{fm}$. Our result for $S^{0}(q, \omega)=-\operatorname{Im~} \Pi^{(0)}(q, \omega) / \pi$ is shown in fig. 1 to- gether with an exact quantum mechanical continuum calculation [4] ${ }^{\ddagger 1}$. Our approach gives practically the exact result for the structureless part of the nuclear response whereas for the low energy part our result still represents very well the average of the exact calculation as expected from our numerous investigations on similar problem [6]. As yet there does not exist an analogous comparison between semiclassical and exact results for the interacting response in the ring approximation. However, as explained in ref. [4], the approximations used to arrive at (1) are exactly of the same nature as in the simple case $(\hbar \rightarrow 0$ limit, local momentum approximation) and therefore we conjecture that the same type of accuracy is obtained in the interacting as in the noninteracting cases (the same arguments hold if a nonlocal mean field is introduced, see below).

In a first step we apply the model of ref. [1] to finite nuclei using our semiclassical theory, that is eq. (1). As in ref. [1] we take the bare nucleon mass and exactly the same force, i.e. $g^{\prime}=0.7$ for the LandauMigdal parameter. In figs. 2, 3a we show the results of this calculation for the transverse structure functions of (e, $\left.\mathrm{e}^{\prime}\right)$ scattering on ${ }^{12} \mathrm{C}$ and ${ }^{48} \mathrm{Ca}$ for the two momentum transfers, $q=2.02 \mathrm{fm}^{-1}$ and $q=1.67 \mathrm{fm}^{-1}$, respectively. We have added to the $1 \mathrm{p}-1 \mathrm{~h}$ response

\footnotetext{
\$1 We are very grateful to N.V. Giai for performing this calculation.
} 


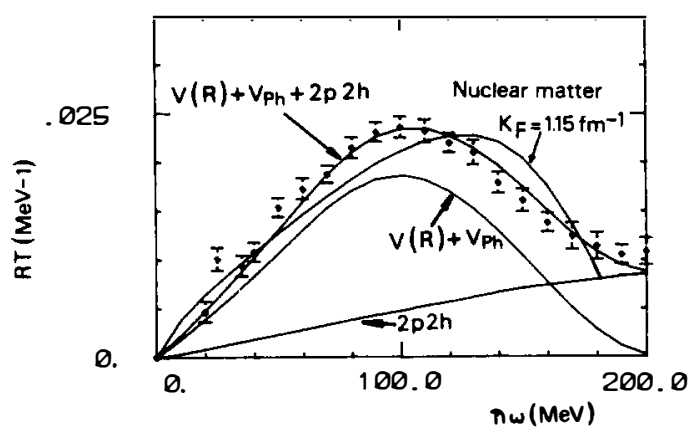

Fig. 2. Transverse nuclear response in ring approximation at $q=2.02 \mathrm{fm}^{-1}$ for ${ }^{12} \mathrm{C}$ in a local potential $V(R)$ [5] with and without $2 \mathrm{p}-2 \mathrm{~h}$ contributions. Also is given the nuclear matter result for $k_{\mathrm{F}}=1.15 \mathrm{fm}^{-1}$ including the $2 \mathrm{p}-2 \mathrm{~h}$ contribution. The data are taken from ref. [7]. The relation between $R_{\mathrm{F}}$ and $S_{\mathrm{T}}$ is given in ref. [1].
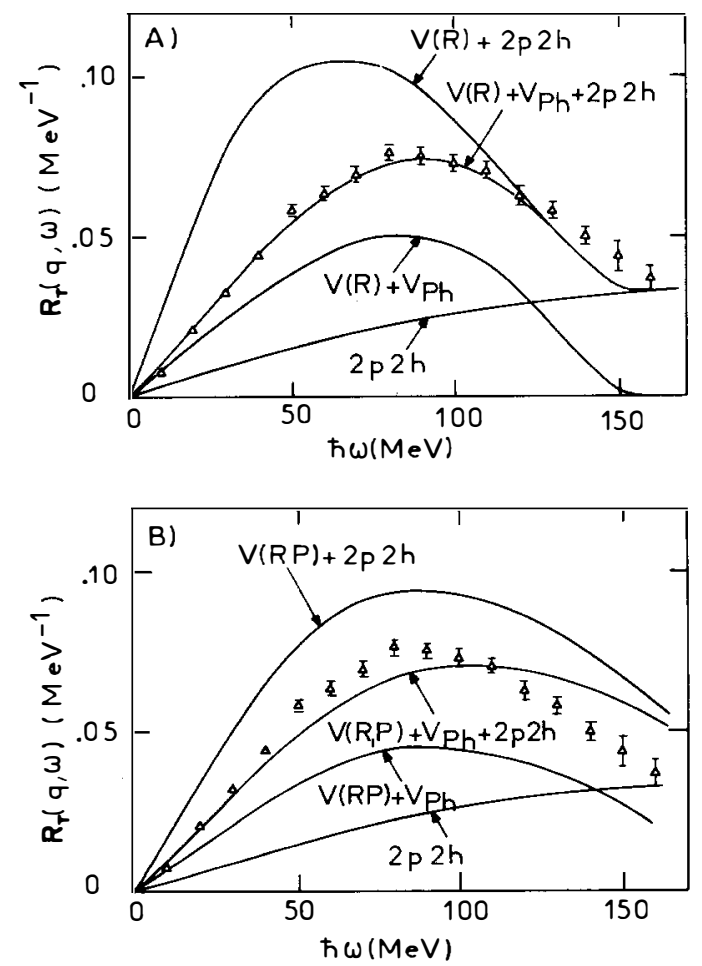

Fig. 3. Transverse nuclear response at $q=1.67 \mathrm{fm}^{-1}$ for ${ }^{48} \mathrm{Ca}$ in two different models (the relation between $R_{\mathrm{T}}$ and $S_{\mathrm{T}}$ is given in ref. [1] ): (a) For a local potential $V(R)$ in ring approximation, with and without $2 \mathrm{p}-2 \mathrm{~h}$ contributions. In addition the free response including $2 \mathrm{p}-2 \mathrm{~h}$ contributions is shown (b) The same as (a) but with a nonlocal potential $V(R, P)$. Experimental points are taken from ref. $[8]^{\neq 2}$.

$\$ 2$ We are grateful to $M$. Bernheim for furnishing his data prior to publication. the $2 \mathrm{p}-2 \mathrm{~h}$ contribution through the ansatz given in ref. [1] which accounts for the filling of the dip. This procedure is justified by the absence of structure in the $2 \mathrm{p}-2 \mathrm{~h}$ part which means that a nuclear matter approach and a semiclassical calculation most likely would show very little difference. We have also incorporated the effect of the $\Delta$-resonance and thus used for the response function $\mathrm{II}^{(0)}=\mathrm{II}_{\mathrm{N}}^{(0)}+\mathrm{II}_{\Delta}^{(0)}[1]$. In a first step we take a universal Landau-Migdal parameter $g^{\prime}$ and later we allow for a difference in the values $g_{\mathrm{NN}}^{\prime}, g_{\mathrm{N} \Delta}^{\prime}, g_{\Delta \Delta}^{\prime}$. In the latter case the expression for the response function reads (in a self evident notation):

$$
\begin{aligned}
\mathrm{II}= & 4 \widetilde{\Pi}_{\Delta}^{(0)}+\left(1+2 V_{\mathrm{N} \Delta} \widetilde{\Pi}_{\Delta}^{(0)}\right)^{2} \mathrm{II}_{\mathrm{N}}^{(0)} \\
& \times\left[1-\Pi_{\mathrm{N}}^{(0)}\left(V_{\mathrm{NN}}+V_{\mathrm{N} \Delta}^{2} \widetilde{\Pi}_{\Delta}^{(0)}\right)\right]^{-1} .
\end{aligned}
$$

Here

$\widetilde{\Pi}_{\Delta}^{(0)}=\mathrm{II}_{\Delta}^{(0)}\left(1-V_{\Delta \Delta} \mathrm{II}_{\Delta}^{(0)}\right)^{-1}$.

As previously stated the above calculations have been performed with the bare nucleon mass. A correct theory should include the renormalisation of the mass coming from the nonlocality of the mean field, giving a typical value $m^{*} / m \simeq 0.7$. For high momentum transfers it is, however, more accurate to take into account the full nonlocality of the mean field. We achieve this by calculating the nonlocal average potential $V^{\mathrm{HF}}\left(k_{\mathrm{F}}, p\right)$ in nuclear matter using the Gogny force [9] and pass again to finite nuclei employing the local momentum approximation. Although the Gogny force for the mean field may seem somewhat inconsistent with the one used for the ph residual interaction [1] we think that this is not too much of a drawback since the Gogny force leads to $m^{*} / m=0.67$ for $k_{\mathrm{F}}=1.35 \mathrm{fm}^{-1}$ which is, as said above, in the range of values any reasonable force should give. The way to calculate the imaginary part of $\mathrm{II}^{(0)}$ to lowest order in $\hbar$ in the presence of a nonlocal mean field has been shown by Rosenfelder [10].

The introduction of the mass renormalisation shifts the end point of $\mathrm{Im} \mathrm{II}^{(0)}$ towards higher energies. It also shifts its peak position to the right and brings the peak down (the integral over $\operatorname{Im} \Pi^{(0)}$ has to be conserved). The nonlocality of the mean field therefore has a similar effect as the residual ph interaction: it quenches and hardens the response. When the $2 \mathrm{p}-2 \mathrm{~h}$ contribution is added the pure Hartree-Fock response is still considerably higher than the experimental one 
as shown in fig. $3 \mathrm{~b}$ indicating the presence of some collectivity. It is, however, clear that with the introduction of the nonlocality of the mean field less collectivity is needed in order to reproduce the experiment, i.e. the Landau-Migdal parameters have to be lowered. An acceptable fit to the experiments is obtained with $g_{\Delta \Delta}^{\prime}$ fixed to the classical Lorentz-Lorenz value currently used for $\pi$ mesic atoms, $g_{\Delta \Delta}^{\prime}=1 / 3$ [11]. The values $g_{\mathrm{NN}}^{\prime}=g_{\mathrm{N} \Delta}^{\prime}=0.6$ then yield the curve shown in fig. $3 \mathrm{~b}$ which is in reasonable agreement with the experimental point of the transverse structure function of ${ }^{48} \mathrm{Ca}$ at $q=1.67 \mathrm{fm}^{-1}$.

We now turn to the longitudinal response in order to interpret the results of the Los Alamos experiment [3] on the ratio of the longitudinal and transverse spin responses probed through proton scattering. In this case the plane wave expression to the excitation operator is invalid since strong absorption prevents the protons to penetrate the nuclear interior. The inclusion of this necessitates the use of optical model wave functions. For high energy protons, however, we can use the eikonal approximation leading to [12]

$\psi_{\mathrm{p}}(r)=\exp \left[\mathrm{i}\left(p z-\frac{p}{2 E} \int_{-\infty}^{z} \mathrm{~d} z^{\prime} U\left(x, y, z^{\prime}\right)\right)\right]$,

with the optical potential

$$
U(r)=V(r)+\mathrm{i} W(r), \quad W(r)=-\frac{1}{2} \hbar \rho(r)(p / m) \sigma(E),
$$

where $\rho(r)=2 k_{\mathrm{F}}^{3}(r) / 3 \pi^{2}$ and $\sigma$ is the total nucleonnucleon cross section. Supposing that the nucleonnucleon interaction is short ranged the excitation operator for the response function then reads:

$\mathrm{e}^{\mathrm{i}\left(\boldsymbol{p}-\boldsymbol{p}^{\prime}\right) r} \rightarrow \psi_{\boldsymbol{p}}(\boldsymbol{r}) \psi_{\boldsymbol{p}^{\prime}}^{*}(\boldsymbol{r})$.

Treating the potentials to lowest order in $\hbar$ (i.e. locally as a constant) we obtain (neglecting the change coming from the real part of $U$ ):

$$
\begin{aligned}
& \Pi_{\mathrm{L}, \mathrm{T}}(q, \omega)=4 \pi \\
& \quad \times \int_{0}^{R_{\mathrm{c}}} \mathrm{d} R R^{2} C(R) \frac{\Pi^{(0)}(R, q, \omega)}{1-v_{\mathrm{L}, \mathrm{T}} \Pi^{(0)}(R, q, \omega)},
\end{aligned}
$$

where the absorption factor $C(R)$ is given by (a similar expression has been given by Bertsch and Scholten [13])

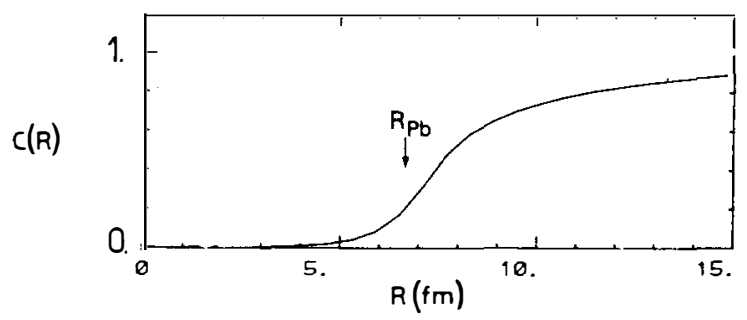

Fig. 4. The surface damping factor as calculated from eq. (6).

$$
\begin{aligned}
& C^{1 / 2}(R)=\frac{1}{2} \\
& \times \int_{-1}^{+1} \mathrm{~d} x \exp \left(-\sigma \int_{-\infty}^{R x} \mathrm{~d} z \rho\left(\left[R^{2}\left(1-x^{2}\right)+z^{2}\right]^{1 / 2}\right)\right)
\end{aligned}
$$

We here have assumed that the inelastic scattering is forward peaked and that the incoming and outgoing proton momenta can approximately be set equal. The function $C(R)$ for $500 \mathrm{MeV}$ protons on ${ }^{208} \mathrm{~Pb}$ is displayed in fig. 4 for $\sigma=30 \mathrm{mb}$.

Finally we show in fig. 5 the corresponding results for $S_{\mathrm{L}}$ and $S_{\mathrm{T}}$ at $q=1.75 \mathrm{fm}^{-1}$ calculated according to eq. (5) with $S_{\mathrm{L}, \mathrm{T}}=-\pi^{-1} \operatorname{Im} \Pi_{\mathrm{L}, \mathrm{T}}$.

No absolute measurements of $S_{\mathrm{L}}$ and $S_{\mathrm{T}}$ have been reported in ref. [3], thus not allowing for an individual comparison. Carey et al. [3] compare the ratio of the longitudinal and transverse responses of ${ }^{208} \mathrm{~Pb}$ to the one of the deuteron, that is $\left[S_{\mathrm{L}} / S_{\mathrm{T}}\right]_{\mathrm{Pb}}$ $\times\left[S_{\mathrm{L}} / S_{\mathrm{T}}\right]_{\mathrm{D}}^{-1}$. The implicit assumption (as in the EMC effect) is that the deuteron behaves as a pair of free nucleons and $S_{\mathrm{L}}=S_{\mathrm{T}}$ for the deuteron. This is

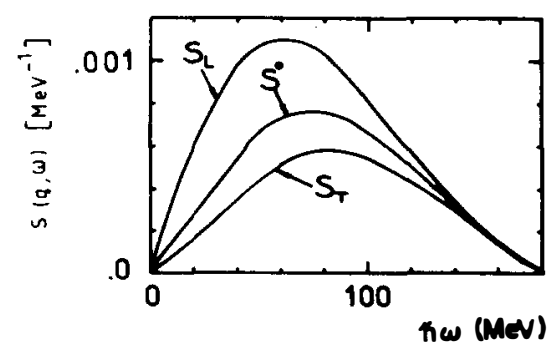

Fig. 5. Longitudinal and transverse nuclear response for $p, p^{\prime}$ scattering together with the free response at $q=1.75 \mathrm{fm}^{-1}$ for ${ }^{208} \mathrm{~Pb}$. Nonlocal mean field and $g_{\mathrm{NN}}^{\prime}=g_{\mathrm{N} \Delta}^{\prime}=0.6, g_{\Delta \Delta}^{\prime}$ $=1 / 3$ have been used. 


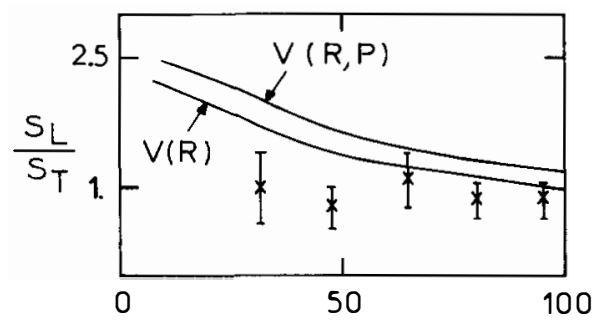

Fig. 6. The contrast $S_{\mathrm{L}} / S_{\mathrm{T}}$ from eq. (3) of ref. [15] at $q$ $=1.75 \mathrm{fm}^{-1}$ for $208 \mathrm{~Pb}$ calculated in a local potential $[V(R)]$ and a nonlocal one $[V(R, P)]$. The experimental points are taken from ref. [3].

not quite the case due to the tensor force: $\left(S_{\mathrm{L}} / S_{\mathrm{T}}\right)_{\mathrm{D}}$ $>1$ and we have applied a small reduction of $\simeq 7 \%$ [14] for our theoretical ratio in order to account for this effect. We also have incorporated the isoscalar contribution through the formula (3) of ref. [15].

For a fixed value of $g^{\prime}$ the ratio is not very sensitive to the introduction of the effective mass. However, as pointed out above, with an effective mass the fit to inelastic electron scattering requires lower $g^{\prime}$ values implying more collectivity in the longitudinal response. This leads in fact to a slight enhancement of the ratio $S_{\mathrm{L}} / S_{\mathrm{T}}$ as shown in fig. 6 .

The experimental points lie below the theoretical curve, in particular those at 30 and $50 \mathrm{MeV}$. The discrepancy with experiment of our results in this work may be attributed to an incorrect treatment of the exchange terms of the ph interaction but also to the fact that for finite nuclei the surface longitudinal and transverse responses do not decouple. Similar results as in this work have been obtained by Bertsch and Esbensen [16], however, without inclusion of the nonlocality of the mean field within the framework of the slab geometry.

In summary we have calculated in a semiclassical formalism the isovector spin responses for finite nuclei. We have found that with a local mean field and with the bare nucleon mass very satisfying fits to the $\left(\mathrm{e}, \mathrm{e}^{\prime}\right)$ data are obtained using by now standard $\mathrm{ph}$ interactions [1]. The introduction of a nonlocal mean field necessitates a reduction of the $g^{\prime}$-values leading to somewhat less collectivity. The resulting agreement with experiment is less satisfactory but still acceptable. The fact that the use of the bare mass allows for superior fits to experiments is intriguing. It could be that a correct treatment of the exchange term of the ph force partially cancels the exchange term of the mean field [17] and we intend to investigate this point.

We also calculated the ratio of the longitudinal to transverse isovector spin response measured in a recent $\left(p, p^{\prime}\right)$ experiment including absorption effects. The ratio is found to be about a factor of two too high at low excitation energies.

\section{References}

[1] W.M. Alberico, M. Ericson and A. Molinari, Nucl. Phys. A 379 (1982) 429; Ann. Phys. (NY) 154 (1984) 356.

[2] E. Oset, H. Toli and W. Weise, Phys. Rep. 83 (1982) 283.

[3] T.A. Carey, K.W. Jones, J.B. McClelland, J.M. Moss, L.B. Rees, N. Tanake and A.D. Bacher, Phys. Rev. Lett. 53 (1984) 144.

[4] U. Stroth, R.W. Hasse and P. Schuck, Proc. Intern. Symp. on Highly excited states and nuclear structure (Orsay, 1983); Proc. Intern. Conf. on Nuclear physics (Florence, 1983); J. Phys. (Paris) Coll. C6 343; P. Schuck, Lecture notes on The random phase approximation (Trieste, February 1984).

[5] W.D. Myers, Nucl. Phys. A145 (1970) 387.

[6] A.H. Blin, M. Durand, R.W. Hasse, B. Hiller and P. Schuck, J. de Phys. (Paris) Coll. C6, 231, 142, 191, 213 and references therein.

[7] P. Barreau et al., Nucl. Phys. A402 (1983) 515; Note CEA N-2334 (1983).

[8] Z.E. Meziani et al., Phys. Rev. Lett. 52 (1984) 2130; 54 (1985) 1233.

We are grateful to $\mathrm{M}$. Bernheim for furnishing the data prior to publication.

[9] U. Gogny and K. Padjen, Nucl. Phys. A293 (1977) 365.

[10] R. Rosenfelder, Ann. Phys. 128 (1980) 188.

[11] M. Ericson and T.E.O. Ericson, Ann. Phys. 36 (1966) 323.

[12] R.G. Newton, Scattering theory of waves and particles (Springer, Berlin, 1982).

[13] G.F. Bertsch and O. Scholten, Phys. Rev. C25 (1982) 804.

[14] M. Ericson and A.W. Thomas, private communication.

[15] W.M. Alberico, M. Ericson and A. Molinari, Phys. Rev. C30 (1984) 1776.

[16] H. Esbensen, H. Toki and G.F. Bertsch, University of Tennessee preprint (1984).

[17] G.F. Bertsch, Phys. Lett. 37B (1972) 470. 